\title{
Testing quantum gravity on extragalactic sources
}

\author{
Aleksandra Piórkowska and Marek Biesiada \\ Department of Astrophysics and Cosmology, Institute of Physics, \\ University of Silesia, Uniwersytecka 4, 40-007 Katowice, Poland \\ E-mail: apiorko@us.edu.pl, marek.biesiada@us.edu.pl
}

In this contribution we raise the issue of how important are the details of cosmological model in the context of testing fundamental questions such like Lorentz invariance violation (LIV) at high energies. We demonstrate that different scenarios explaining present accelerating expansion of the Universe even though best fitted to observations yet give different predictions for the time of flight delays suggested by some approaches to quantum gravity. Moreover, we show that certain tendency in energy dependent time of flight delays reported in gamma-ray bursts under assumption of LambdaCDM model is also present in alternative cosmological scenarios. It turns out to be the most pronounced in cosmology with time variable dark energy equation of state.

Next result presented in this contribution is a new proposal of using energy dependent time delays between images of gravitationally lensed source. Having developed an idea that details of cosmological model do matter for some tests of fundamental physics, we go on to enrich the existing tests with a new one, namely that using strong gravitational lensing.

25th Texas Symposium on Relativistic Astrophysics - TEXAS 2010

December 06-10, 2010

Heidelberg, Germany 


\section{Introduction}

Modern approaches to quantum gravity predict spacetime with non-trivial properties at high energies or small scales. This can be reflected in breaking of basic symmetries like Lorentz symmetry. In particular, specific strategy that enjoys great popularity and which has been recently extensively studied, is phenomenology based on modification of dispersion relation of the relativistic particles. Such approach is perhaps the simplest framework for Lorentz symmetry violation (LIV). It postulates that the usual Lorentz invariant dispersion law should be replaced by

$$
E^{2}=p^{2} c^{2}+m^{2} c^{4}+\varepsilon E^{2}\left(\frac{E}{\xi_{n} E_{Q G}}\right)^{n}
$$

The 'sign parameter' $\varepsilon= \pm 1$ corresponds to super or infraluminal motion of a particle and $\xi_{n}$ is a dimensionless parameter related to the magnitude of LIV with $n=1,2, \ldots$ - the order of a correction to ordinary, Lorentz invariant case. $E_{Q G}$ is quantum gravity energy scale (energy scale at which the effects associated with quantum gravity becomes visible) often assumed to be the Planck energy scale. $H(z)$ is cosmological expansion rate known as the Hubble function. An extra term on the right hand side of (1.1) introduces violation of boost symmetry in model independent way. This approach may seem superficial (lack of dynamics and deeper analysis), but in its simplicity is very useful from the experimental point of view: even small deviation from Lorentz invariance (which holds perfectly at low energies) should be noted as a new effect observed in the propagation of ultra-relativistic particles.

The relation (1.1) leads to many non-standard effects. Among other implications it makes the group velocity of relativistic particles and thus, the time of flight from distant source (of redshift $\mathrm{z}$ ) to the Earth, energy dependent:

$$
\begin{gathered}
v(t)=\frac{\partial H}{\partial p} \simeq c(1+z)\left[1-\frac{1}{2} \frac{m^{2} c^{4}}{E_{0}^{2}(1+z)^{2}}+\varepsilon \frac{(n+1)}{2}\left(\frac{E_{0}}{\xi_{n} E_{Q G}}\right)^{n}(1+z)^{n}\right] \\
t=\frac{1}{c} \int_{t_{\text {emission }}}^{t_{0}} v(t) d t=\int_{0}^{z}\left[1-\frac{m^{2} c^{4}}{2 E_{0}} \frac{1}{\left(1+z^{\prime}\right)^{2}}+\varepsilon \frac{n+1}{2}\left(\frac{E_{0}}{\xi_{n} E_{Q G}}\right)^{n}\left(1+z^{\prime}\right)^{n}\right] \frac{d z^{\prime}}{H\left(z^{\prime}\right)} .
\end{gathered}
$$

So, time delay due to both, particle masses and LIV effects, between a high energy particle and a low energy prompt photon is equal to:

$$
\Delta t=\int_{0}^{z}\left[\frac{m^{2} c^{4}}{2 E_{0}} \frac{1}{\left(1+z^{\prime}\right)^{2}}-\varepsilon \frac{n+1}{2}\left(\frac{E_{0}}{\xi_{n} E_{Q G}}\right)^{n}\left(1+z^{\prime}\right)^{n}\right] \frac{d z^{\prime}}{H\left(z^{\prime}\right)} .
$$

In particular, considering low energy and high energy photons with energy difference $\Delta E$, the above formula (for $n=1$ ) reduces to

$$
\Delta t=\frac{\Delta E}{E_{Q G}} \int_{0}^{z} \frac{\left(1+z^{\prime}\right) d z^{\prime}}{H\left(z^{\prime}\right)} .
$$

From the above formula one can easily find that, among many extremely precise tests that have yielded the most important bounds on LIV, time delay technique deserves special attention in the context of high energy astroparticle physics: to put any constraints on quantum gravity one can use astrophysical objects like pulsars or active galactic nuclei (AGNs), especially blazars (BL Lac) to look for energy dependent differences in times of arival of photons. This is because one needs a 
signal with appriopriate time structure (variability of order of milli-second and better), hard energy spectrum (more than $20 \mathrm{MeV}$ ) and cosmological distances (non-standard effects can accumulate while particle propagates itself through a spacetime with LIV properties). Specifically, gamma ray bursts (GRBs) being highly energetic events visible from cosmological distances are the most promising sources of constraining LIV theories.

However, the idea of searching for time of flight delays is tempered by some effects that prevent obtaining better accuracy. Firstly, the Universe filled with $2.7 \mathrm{~K}$ cosmic microwave background radiation becomes opaque, via pair production process, to photons with energies above 10 $\mathrm{TeV}$ (analogous to GZK threshold for particles). Despite the fact that $20 \mathrm{TeV}$ photons were reported from Mk 501 BL Lac object [2], the use of very high energy photons from GRBs can be tricky). Secondly, time delay analysis strongly depends on intrinsic delay (at source frame) in different energy channels [3]. This clearly disfavors using energy dependent patterns in time-of-flights from single sources. Hopefully, method of measuring time of flight is a subject of continuous improvement. Few years ago Jacob and Piran proposed to use high energy neutrinos instead of photons [5] to overcome the problem with pair production. Emission of $10^{2}-10^{4} \mathrm{TeV}$ neutrinos is typically predicted in current models of GRBs [4] and as noticed in [5] neutrino detectors like Ice Cube should be extremely quiet in this energy range. So, measurements of time delay between prompt gamma ray photons and neutrino signal would open a new window on exploring LIV theories. As a possible way out to the intrinsic time-lags problem Ellis et al. ([3], see also [6]) proposed to work on a statistical ensamble of GRBs by using linear regression analysis where the intercept represents intrinsic time delay and the linear term represents LIV effect. As a result of such analysis they found a weak evidence for LIV and therefore the idea of time delay measurements is worth further consideration.

\section{LIV induced time delays in different cosmological models}

The discovery of accelerated expansion of the Universe introduced the problem of dark energy which is now one of the most important issues in modern cosmology. The so called $\Lambda \mathrm{CDM}$ model is a standard assumption in most of the considerations concerning modern physics, also in time delay analysis. This model fits rather well to independent data (such like CMBR, LSS and supernovae data) but suffers from cosmological constant fine tuning problem (being constant, why does it start dominating at the present epoch?) and from the enormous discrepancy between facts and expectations (assuming that Lambda represents quantum-mechanical energy of the vacuum it should be 55 orders of magnitude larger than observed). Apart from $\Lambda$ CDM model, a lot of specific scenarios have been put forward as an explanation of dark energy puzzling phenomenon. They fall into two broad categories: hypothetical material candidates for dark energy (cosmological constant $\Lambda$, quintessence - evolving scalar fields, Chaplygin gas or modification of gravity theory (e.g. brane world scenarios).

According to fact that GRBs are at cosmological distances, the details of cosmological model should not be negligible. To see, how strong could be such effect and following [5] for better comparison of results we calculated time delay of $100 \mathrm{TeV}$ neutrinos with respect to the low energy prompt photon signal (we used (1.4 in our calculations) in five different cosmological scenarios already mentioned with parameters best fitted to current data. Expansion rates and the values of 


\begin{tabular}{|c|c|c|}
\hline Model & Cosmological expansion rate $H(z)$ & Parameters \\
\hline$\Lambda$ CDM & $H^{2}(z)=H_{0}^{2}\left[\Omega_{m}(1+z)^{3}+\Omega_{\Lambda}\right]$ & $\Omega_{m}=0.3$ and $\Omega_{\Lambda}=0.7$ \\
Quintessence & $H^{2}(z)=H_{0}^{2}\left[\Omega_{m}(1+z)^{3}+\Omega_{Q}(1+z)^{3(1+w)}\right]$ & $w=-0.87$ \\
Var Quintessence & $H^{2}(z)=H_{0}^{2}\left[\Omega_{m}(1+z)^{3}+\Omega_{Q}(1+z)^{3\left(1+w_{0}-w_{1}\right)} \exp \left(3 w_{1} z\right)\right]$ & $w_{0}=-1.5$ and $w_{1}=2.1$ \\
Chaplygin Gas & $H(z)^{2}=H_{0}^{2}\left[\Omega_{m}(1+z)^{3}+\Omega_{C h}\left(A_{0}+\left(1-A_{0}\right)(1+z)^{3(1+\alpha)}\right)^{\frac{1}{1+\alpha}}\right]$ & $\alpha=1$ and $A_{0}=0.83$ \\
Braneworld & $H(z)^{2}=H_{0}^{2}\left[\left(\sqrt{\Omega_{m}(1+z)^{3}+\Omega_{r_{c}}}+\sqrt{\Omega_{r_{c}}}\right)^{2}\right]$ & $r_{c}=1.4 H_{0}^{-1}$ and $\Omega_{r_{c}}=\frac{1}{4}\left(1-\Omega_{m}\right)^{2}$ \\
\hline
\end{tabular}

Table 1: Expansion rates $H(z)$ and values of the parameters (best fitted to SNIa and CMBR data) in four models tested.
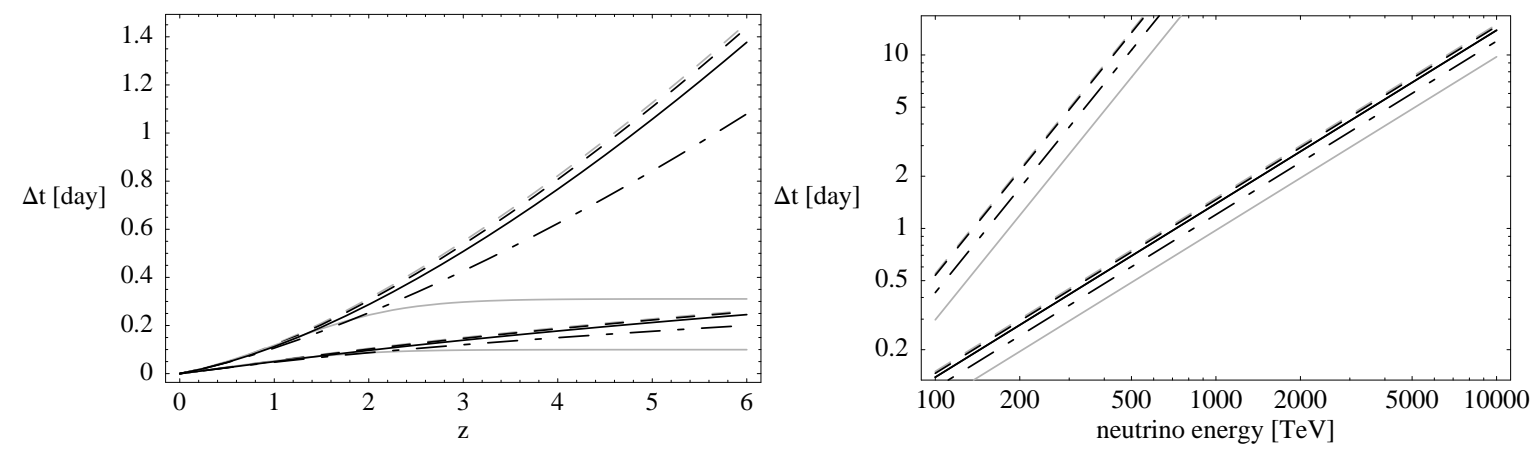

Figure 1: Observed time delays for $100 \mathrm{TeV}$ neutrinos as a function of redshift (left panel) and neutrino energy (right panel) in different dark energy scenarios ( $\Lambda \mathrm{CDM}$ - light gray dashed line, quintessence black dashed line, quintessence with varying E.O.S. — light gray solid line, brane world model — black solid line and Chaplygin gas scenario - dot-dashed line). Upper curves correspond to $n=2, \xi_{2}=10^{-7}$, lower curves correspond to $n=1, \xi_{1}=1$.

parameters are summarised in Table 1. In order to get the results comparable with [5] we assumed $E_{Q G}$ equal to the Planck energy, $\xi_{1}=1$ and $\xi_{2}=10^{-7}$ for $n=1$ and $n=2$ case respectively. In our calculations we retained the neutrino mass - it is massive after all - and we assumed $m_{v}=1 \mathrm{eV}$. However it is evident already from the formula (1.4) that the effect of non-zero mass of the neutrino is for our purpose negligible - in perfect accordance with formulas in [5]. Our results are plotted together in Figure 1.

\section{Statistical analysis of gamma ray burst data: in search for LIV signals in different cosmological models}

The observational strategy emerging from equation (1.5) is very simple: monitor appropriate (i.e. emitting both low and high energy photons) cosmological source at different energy channels and try to detect this time delay. However there remains an indispensable uncertainty: there is no reason for which low and high energy signal should be emitted simultaneously, and while detecting distinct signals (peaks in the light curve) at different energies we have no idea which one was sent first. This is known as so-called intrinsic time lags problem. In [3] it has been noticed that while this ambiguity clearly disfavors using energy dependent patterns in time-of-flights from single sources, 


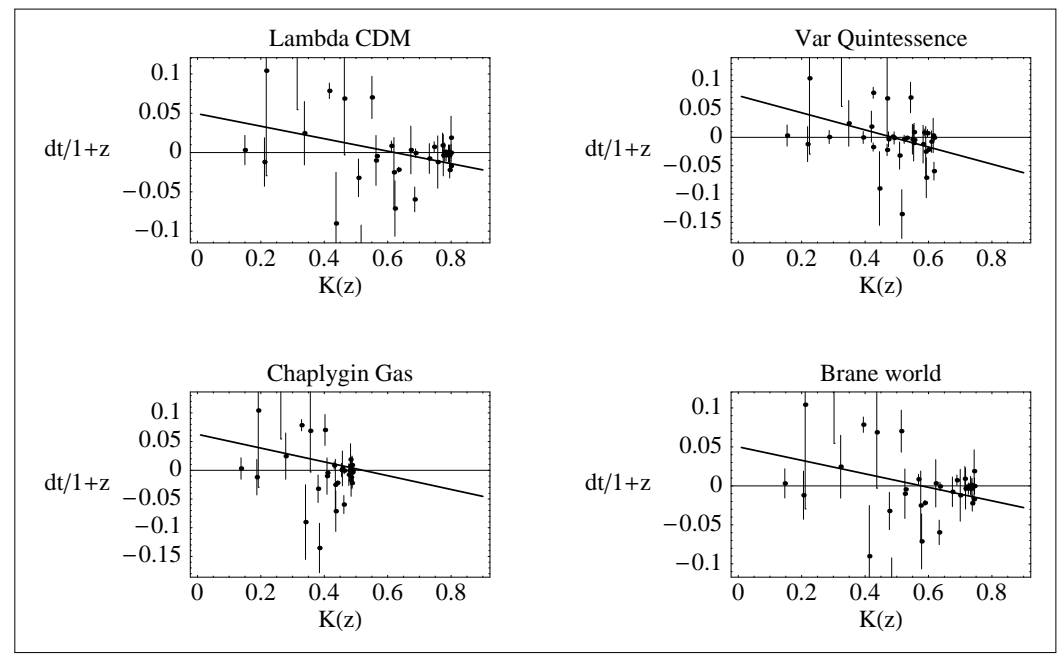

Figure 2: Results of the linear regression for time delay vs $\mathrm{K}(\mathrm{z})$ technique in the cosmological models tested (the case of Quintessence was too similar to the $\Lambda \mathrm{CDM}$ to deserve displaying).

one is still able to search for statistical correlations of spectral time lags with redshift in an ensamble of sources located at different redshifts. If one decomposes the observed time delay $\Delta t_{o b s}$ between different energy channels: $\Delta t_{o b s}=\Delta t_{L I V}+\Delta t_{\text {intrinsic }}$ then, using the notation of [3], after taking into account cosmological time dilation factor $1+z$, one has $\Delta t_{o b s}=a_{L I V}(1+z) K+b(1+z)$, where: $K=\frac{1}{1+z} \int_{0}^{z} \frac{\left(1+z^{\prime}\right) d z^{\prime}}{h\left(z^{\prime}\right)}$ and $a_{L I V}=\frac{\Delta E}{H_{0} E_{Q G}}$. Such parametrization allows to formulate the problem in terms of linear regression:

$$
\frac{\Delta t_{o b s}}{1+z}=a_{L I V} K(z)+b
$$

where the intercept informs about intrinsic time lags, and slope carries information about LIV effects. Following this line Ellis et al. ([2,3]) used a sample of gamma-ray bursts (GRBs) with known redshifts. In original paper by Ellis et al. ([3]) there were some hints towards the energy-dependent time delays in the gamma-ray bursts. However, this result was obtained within a concordance cosmological model. In order to see how this method performs in alternative cosmological scenarios, we have tested it in five classes of models described in the previous section.

The sample we used consists of 35 GRBs with known redshifts for which time lags between different energy channels have been assessed from the light curves by Ellis et al. [3]. The data are based on the results of BATSE, HETE and Swift experiments. Technical details can be found in [3]. We took these data for the sake of comparability. Results are summarised in the Figure 2. One can see that in all classes of alternative cosmological models the effect is similar, hence there is no indication that Ellis et al. result might be an artifact of assuming $\Lambda \mathrm{CDM}$.

\section{Gravitational lensing time delays as a tool for testing quantum gravity phenomenology}

Gravitational lensing of quasars and extragalactic radio sources at high redshifts by foreground galaxies is now well established and has developed into a mature branch of both theoretical and 
observational astrophysics. Misalignment of the source, the lens and observer results typically in multiple images whose angular positions and magnification ratios allow reconstructing lensing mass distribution. In particular they provide an independent confirmation of dark matter in galaxies and became an important tool for investigating dark matter distribution. Another important ingredient of gravitational lensing is the time delay between lensed images of the source. This effect originates as a competition between Shapiro time delay from the gravitational field and the geometric delay due to bending the light rays and is best understood in terms of Fermat principle. In other words, the intervening mass between the source and the observer introduces an effective index of refraction, thereby increasing the light travel time.

The lensing is called strong if source position happens to lie within the so called Einstein ring - the circle of a radius $\vartheta_{E}$ (defining the proper deflection scale of a given lens). The Einstein ring radius for the singular isothermal sphere (SIS) model, surprisingly realistic model of the lens potential, is: $\vartheta_{E}=4 \pi \frac{D_{l s}}{D_{s}} \frac{\sigma^{2}}{c^{2}}$, where $\sigma$ denotes one-dimensional velocity dispersion of stars in lensing galaxy. If the lensing is strong then two co-linear images $A$ and $B$ form on the opposite side of the lens, at radial distances $R_{A}=\beta+\vartheta_{E}$ and $R_{B}=\vartheta_{E}-\beta\left(\beta<\vartheta_{E}\right)$ having time delays between the images:

$$
\Delta t_{S I S}=\frac{1+z_{l}}{2 c} \frac{D_{l} D_{s}}{D_{l s}}\left(R_{A}^{2}-R_{B}^{2}\right)=\frac{2\left(1+z_{l}\right)}{c} \frac{D_{l} D_{s}}{D_{l s}} \vartheta_{E} \beta=\frac{8 \pi}{H_{0}} \widetilde{r}_{l} \beta \frac{\sigma^{2}}{c^{2}}
$$

$D_{l}, D_{s}$ are angular diameter distances to the lens and the source located at redshifts $z_{l}$ and $z_{s}$ respectively ( $D_{l s}$ is the angular diameter distance between lens and source); $\tilde{r}_{l}$ denotes the reduced comoving distance (i.e. a comoving distance expressed as a fraction of the Hubble horizon $d_{H}=$ $c / H_{0}$ ) to the lens. The most right hand part of the equation (4.1) is more useful from the theoretical point of view. In particular it shows explicitly that the time delay between images is created at the lens location ( $\widetilde{r}_{l}$ factor). This time delay is achromatic in general relativity.

Let us now imagine a source at cosmological distance emitting low energy and high energy (in $\mathrm{TeV}$ range) photons which undergoes gravitational lensing by a foreground galaxy. Let us also assume that LIV type distorted dispersion relation (1.1) holds. The observer would also notice time delays between images, but this time it would be a combined effect of gravitational lensing and LIV. Therefore it would no longer be achromatic:

$$
\Delta t_{L I V, S I S}=\frac{8 \pi}{H_{0}} \widetilde{r}_{L I V}\left(z_{l}\right) \beta \frac{\sigma^{2}}{c^{2}}, \quad \widetilde{r}_{L I V}\left(z_{l}\right)=\widetilde{r}_{l}+H_{0} \frac{n+1}{2}\left(\frac{E}{\xi_{n} E_{Q G}}\right)^{n} \int_{0}^{z_{l}} \frac{\left(1+z^{\prime}\right)^{n} d z^{\prime}}{H\left(z^{\prime}\right)} .
$$

Because the LIV effect is expected to be extremely small, let us restrict further to the $n=1$ case.

Now we can assume that observations in low energy would essentially provide time delay between images equal to $\Delta t_{S I S}$, whereas monitoring of the same images in high energy $(\mathrm{TeV})$ channel would provide $\Delta t_{L I V, S I S}$. These two measurements would differ by

$$
\Delta t_{L I V, S I S}-\Delta t_{S I S}=\frac{8 \pi}{H_{0}} \beta \frac{\sigma^{2}}{c^{2}} \frac{E}{E_{Q G}} \int_{0}^{z} \frac{\left(1+z^{\prime}\right) d z^{\prime}}{H\left(z^{\prime}\right)} .
$$

This opens up a possibility to study time delays induced by LIV. Namely, monitoring the time delays between lensed images performed in different energy channels (e.g. optical or gamma-ray - low energy and $\mathrm{TeV}$ - high energy photons) may reveal extra delays due to distorted dispersion relation typical in LIV theories. This test is free from the systematics inherent in other settings. 


\section{Summary and conclusions}

In this contribution we have discussed the sensitivity of time delay technique to the details of cosmological model.In particular, it has been shown that lack of detailed knowledge about cosmological model (in the context of accelerating expansion of the Universe) can be another source of systematic effects at high redshifts. Following this line, we have extended linear regression analysis of intrinsic time-lags considering alternative models of 'dark energy'. The result is that the effect noticed by Ellis et al. is also present in those models and is strongest in one of them (quintessence model with variable equation of state). We have also proposed a new test based on gravitational lensing by monitoring time delays between images of gravitationally lensed quasars in low and high energy channels. In standard theory (general relativity) the result should be the same - gravitational lensing is essentially achromatic. On the other hand in the presence of LIV effects time delays loose this property - high energy photons should come at different times comparing with low energy ones. Therefore time delays between images should be different at different energies. Because this method is differential in nature, it gets rid of the assumptions about intrinsic time delays of signals at different energies. Since the time delay between images is produced at the lens location, the result does not depend very strongly on the cosmological model.

This contribution was supported by the Polish Ministry of Science Grant no N N203 390034.

\section{References}

[1] G. Amelino-Camelia at al., Potential Sensitivity of Gamma-Ray Burster Observations to Wave Dispersion in Vacuo, Nature 393 (763) 1998 [arXiv:astro-ph/9712103v2].

[2] J. Albert et al., Probing Quantum Gravity using Photons from a Mkn 501 Flare Observed by MAGIC, Phys. Lett. B 668 (253) 2008 [arXiv:0708.2889].

[3] J. Ellis et al., Robust Limits on Lorentz Violation from Gamma-Ray Bursts, Astropart. Phys. 25 (402) 2006 [arXiv:astro-ph/0510172].

[4] T. Piran, The physics of gamma-ray bursts, Rev. Mod. Phys. 76 (1143) 2005.

[5] U. Jacob and T. Piran, GRBs neutrinos as a tool to explore quantum gravity induced Lorenz violation, Nature Phys. 3 (87) 2007 [arXiv:hep-ph/0607145v1].

[6] U. Jacob and T. Piran, Lorentz-violation-induced arrival delays of cosmological particles, J. Cosmol. Astropart. Phys. 0801 (031) 2008 [arXiv:0712.2170]; J.R. Ellis et al., Erratum (astro-ph/0510172): Robust Limits on Lorentz Violation from Gamma-Ray Bursts, [arXiv:0712.2781].

[7] M. Biesiada and A. Piórkowska, GRB neutrinos, Lorenz Invariance Violation and the influence of background cosmology, J. Cosmol. Astropart. Phys. 0705 (011) 2007 [arXiv:0712.0937].

[8] M. Biesiada and A. Piórkowska, Lorentz Invariance Violation induced time delays in GRBs in different cosmological models, Class. Quantum Grav. 26 (125007) 2009 [arXiv:1008.2615].

[9] M. Biesiada and A. Piórkowska, Gravitational lensing time delays as a tool for testing Lorentz Invariance Violation, Mon. Not. R. Astronom. Soc. 396 (946-950) 2009 [arXiv:0712.0941]. 